TITLE:

\title{
Macrocyclic oligoarylamine-based spin system
}

$\operatorname{AUTHOR}(\mathrm{S})$ :

Ito, Akihiro; Tanaka, Kazuyoshi

\section{CITATION:}

Ito, Akihiro ...[et al]. Macrocyclic oligoarylamine-based spin system. Pure and Applied Chemistry 2010, 82(4): 979-989

ISSUE DATE:

2010

URL:

http://hdl.handle.net/2433/148418

RIGHT:

Copyright (C) 2009 International Union of Pure and Applied Chemistry 
Pure Appl. Chem., Vol. 82, No. 4, pp. 979-989, 2010.

doi:10.1351/PAC-CON-09-10-16

(C) 2010 IUPAC, Publication date (Web): 17 March 2010

\title{
Macrocyclic oligoarylamine-based spin system*
}

\author{
Akihiro Ito and Kazuyoshi Tanaka ${ }^{\ddagger}$ \\ Department of Molecular Engineering, Kyoto University, Nishikyo-ku, Kyoto \\ 615-8510, Japan
}

\begin{abstract}
Alternating meta- and para-phenylene-linked oligoarylamines are considered as promising molecular parts for the molecule-based electronics and/or spintronics due to their intriguing electronic and magnetic properties. From the magnetic viewpoint, a metaphenylene linker plays a crucial role in ensuring the effective ferromagnetic interaction, while para-phenylene linker plays an important part in stabilizing the spin-containing aminium radical cations. Of the meta-para and all-meta oligoarylamines prepared so far, the macrocyclic oligoarylamines are structurally defined, and therefore we can employ them as the component pieces to prepare the two- or three-dimensionally structured oligoarylamines. The spin electronic properties of polycationic species generated from two kinds of macrocyclic oligoarylamines, which will be able to be extended into the $2 \mathrm{D}$ multi-spin molecular systems, are described on the basis of the pulsed electron spin resonance (ESR) measurements.
\end{abstract}

Keywords: arylamines; multi-redox activity; poly(radical cation)s; 2D multi-spin molecular systems.

\section{INTRODUCTION}

High-spin organic polymers have attracted much attention since the famous prediction of Mataga and Itoh [1]. In particular, the 2D network structure shown in Fig. 1a is quite interesting in that the thermodynamic instability inherent in 1D multi-spin chain structure [2] might be solved by 2D multi-spin network structure and that the rigid structure would stabilize the radical centers that are often toward interand intramolecular recombination reactions. In this context, macrocyclic multi-spin molecular systems are regarded as potential building blocks for hypothetical covalent 2D multi-spin polymers and therefore some attempts to synthesize macrocyclic multi-radical systems have been already reported [3].

Another critical point for construction of $2 \mathrm{D}$ multi-spin polymers is curvature. The ChemDraw representation shown in Fig. 1a gives the impression that the Mataga-Itoh polymer has a planer conformation. However, the steric hindrance among the substituted hydrogen atoms does not allow such a planar conformation. Instead, the resulting curvature causes a bowl-like structure. Such a structure would be interesting from the viewpoint of curved $\pi$-conjugation, which has been often been discussed in fullerene chemistry [4]. However, the curvature causes problems in achieving globally flat 2D polymers.

For example, Rajca and co-workers succeeded in the generation of a spin-quintet state of a metacyclophane compound (Fig. 1b) based on the triarylmethyl radical at low temperatures [5]. On the other hand, Iwamura and co-workers reported the generation of a spin-tridecet metacyclophane (Fig. 1c) by

*Pure Appl. Chem. 82, 757-1063 (2010). An issue of reviews and research papers based on lectures presented at the $13^{\text {th }}$ International Symposium on Novel Aromatic Compounds (ISNA-13), 19-24 July 2009, Luxembourg City, Luxembourg on the theme of aromaticity.

¥Corresponding author 
(a)

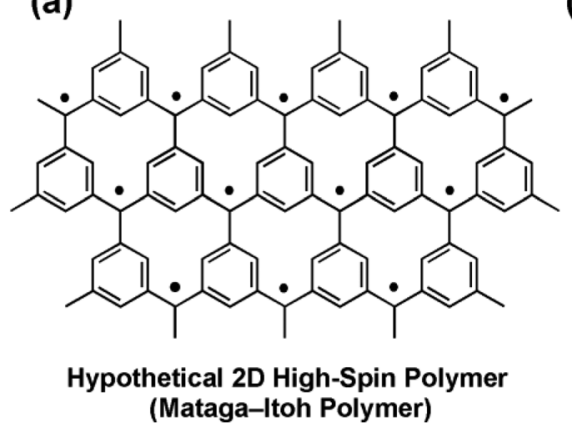

(b)

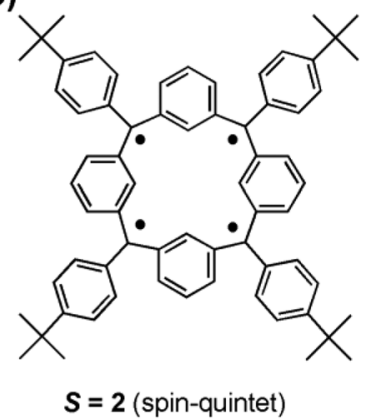

(c)

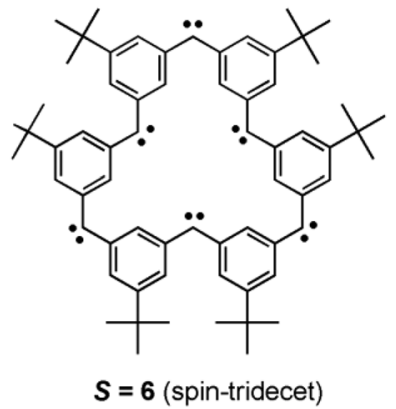

Fig. 1 Carbon-centered multi-radical macrocyclic molecular systems.

photolysis of the macrocyclic hexadiazo compound at cryogenic temperartures [6]. However, unfortunately, these high-spin species were still highly reactive at elevated temperatures.

Highly reactive carbon-centered radicals can be replaced with relatively stable nitrogen-centered radicals (Fig. 2). We have investigated nitrogen-centered polyradical systems (Fig. 2b) through direct polymerization or oligomer model studies of arylamines [7]. The reason stems from (i) the multi-redox activity of oligoarylamines and (ii) the relative stability of generated poly(radical cation)s, and furthermore, (iii) the recent development of Pd-catalyzed aryl amination reactions facilitating the synthesis of oligoarylamines with complicated structures [8]. In addition, Janssen and co-workers demonstrated that the high-spin molecules composed of the semi-quinoid radical cation of para-phenylenediamine (PD) moiety (Fig. 2c) are far more stable than those of the aminium radical cations. Hence, high-spin molecular systems based on the 1D or dendritic oligoarylamines have been extensively examined $[9,10]$.

(a)

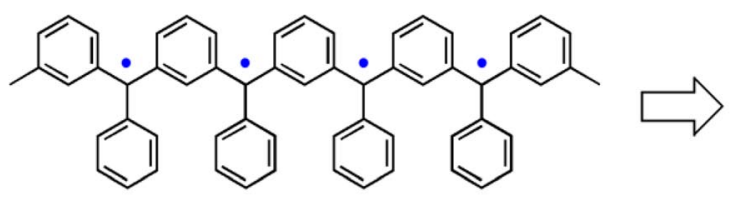

(b)

Carbon-centered multi-radical<smiles>Cc1cccc([NH+](c2ccccc2)c2cccc([NH+](c3cccc([NH+](c4ccccc4)c4ccccc4)c3)c3cccc([NH+](c4ccccc4)c4ccccc4)c3)c2)c1</smiles><smiles>Cc1cccc(N(c2ccccc2)c2ccc(N(c3ccccc3)c3cccc(N(c4ccccc4)c4cccc(N(c5ccccc5)c5ccccc5)c4)c3)cc2)c1</smiles>

Nitrogen-centered multi-radical

Fig. 2 From the carbon-centered multi-radicals to the nitrogen-centered multi-radicals.

With the aforementioned points in mind, as shown in Fig. 3, we are interested in hypothetical 2D macrocyclic oligoarylamine-based polymers which may lead to the globally flat 2D network multi-spin systems.

In particular, the ground state for the oxidized 2D polymer shown in Fig. 3b was theoretically predicted to be a high-spin state [11]. As the first step, we examined the spin electronic properties of two kinds of macrocycles shown in blue in Fig. 3 as building blocks of these 2D polymers $[12,13]$. 


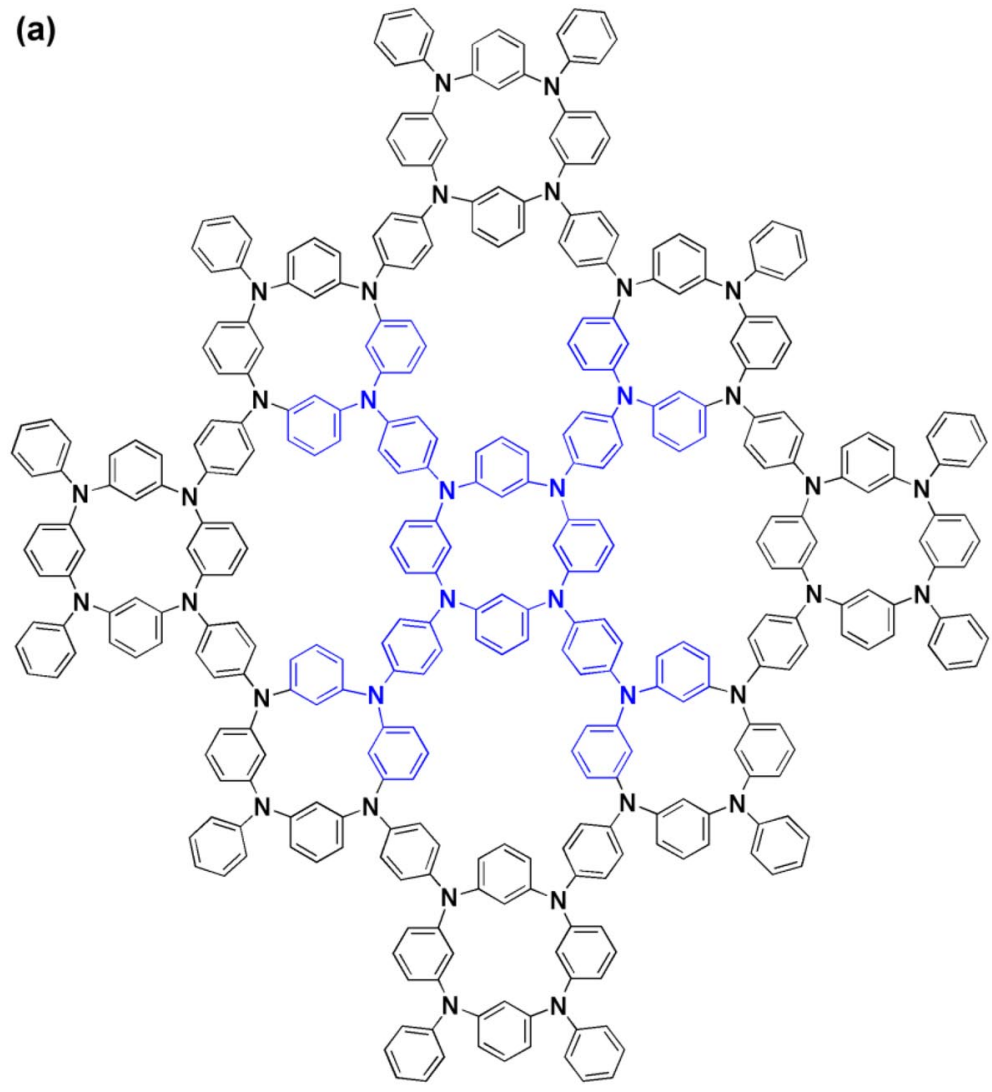

(b)

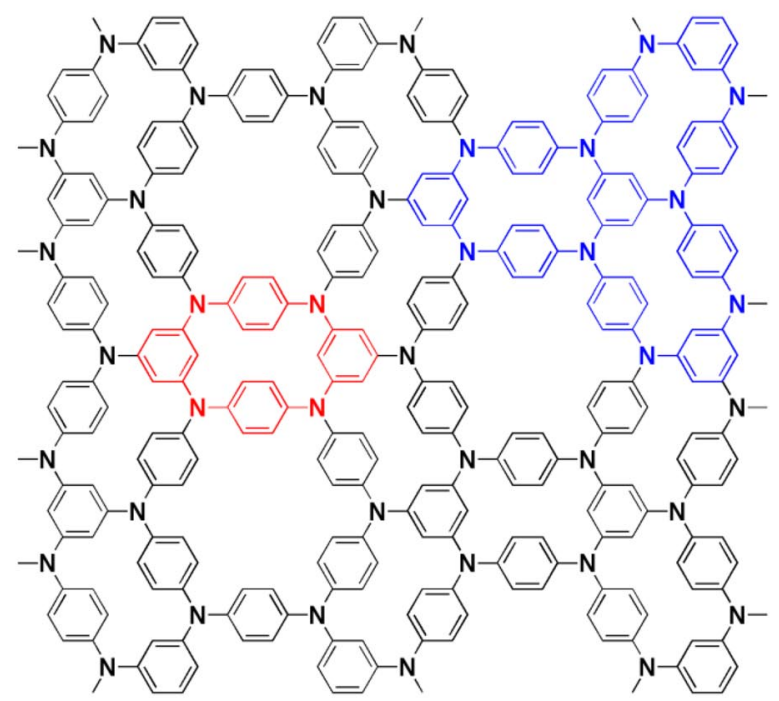

Fig. 3 Hypothetical 2D polymers based on macrocyclic oligoarylamines: (a) The polymer is comprised of tetraaza $\left[1_{4}\right]$ metacyclophene macrocyclic parts and para-phenylene linkers. 4-( $N, N$-Diphenylamino)phenylsubstituted tetraaza[ $\left.11_{4}\right]$ metacyclophene is colored in blue, and is considered as a constitutional unit; (b) The polymer is comprised of tetraaza $\left[1_{4}\right] m, p, m, p$-cyclophane macrocyclic parts, which is colored in red. The bluecolored trimacrocyclic moiety is regarded as one of the interesting fragments quarried out from this polymer. 


\section{RESULTS AND DISCUSSION}

\section{Synthesis of target molecules}

The target molecules have been prepared from corresponding arylamines and aryl halides by utilizing the Pd-catalyzed aryl amination reaction (Buchwald-Hartwig reaction) [8]. The yield in each reaction is comparatively low, but it is intriguing that the complicated structures are constructed in a one-pot manner (Fig. 4, Supplementary data).

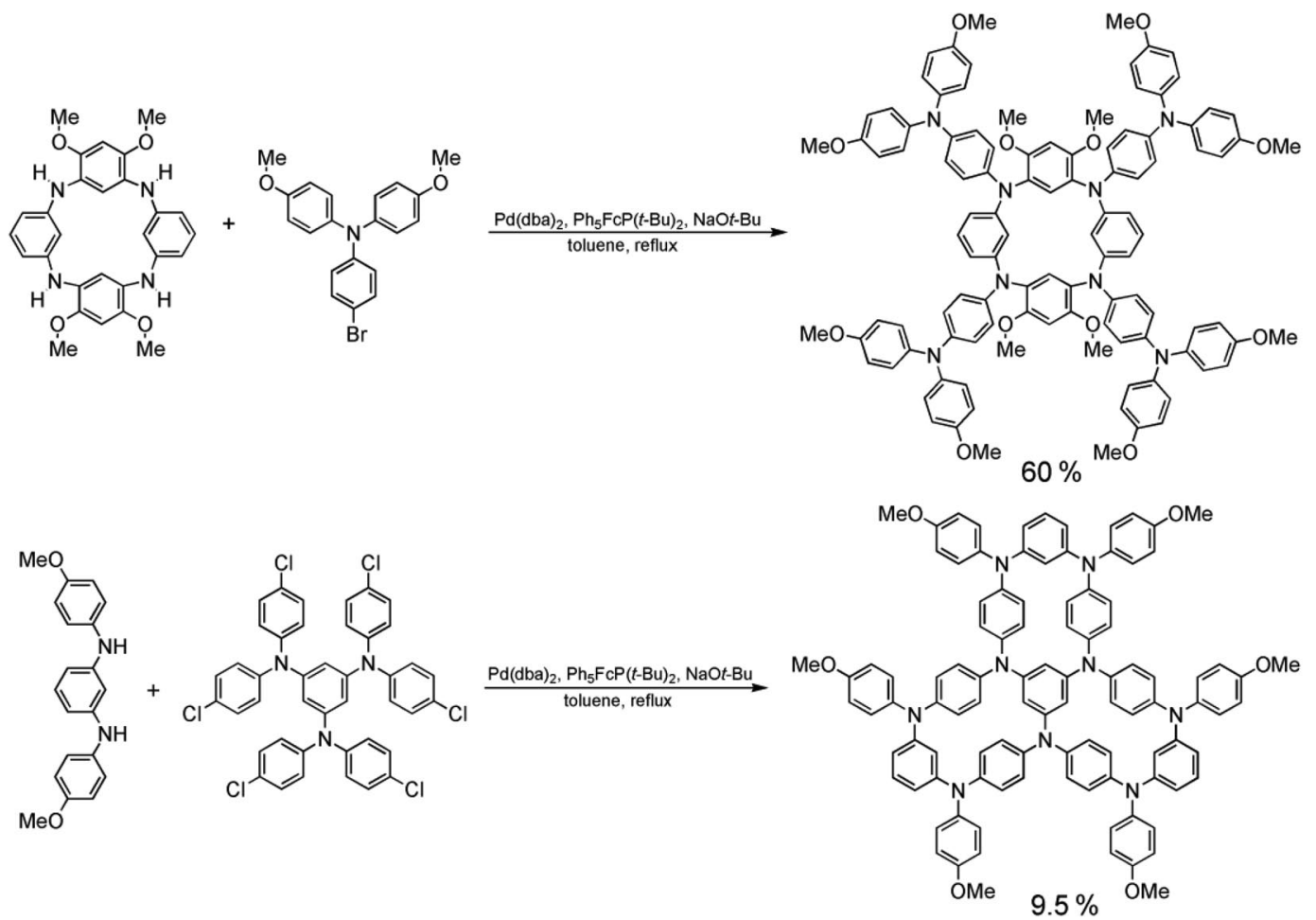

Fig. 4 Synthetic routes of target molecules studied here.

\section{Tetraaza[ $\left.\mathbf{1}_{4}\right]$ metacyclophane}

Tetraaza[1 $\left.1_{4}\right]$ metacyclophane was first reported by Smith in 1963 [14,15] (Fig. 5). However, his report contained no detailed description of the synthetic procedure, physicochemical properties, and so forth. About 36 years later since this report, we have succeeded in the preparation of a series of $N$-methylsubstituted azametacyclophanes including tetraaza[1 $\left.1_{4}\right]$ metacyclophane 1 to realize the toroidal highspin molecular systems [16]. However, we encountered the difficulty that we were not able to generate the monocation of $\mathbf{1}$, let alone the tetracation of $\mathbf{1}$.

The first oxidation process of $\mathbf{1}$ was found to be irreversible by cyclic voltammetry, and, therefore, the generated monocation decomposes immediately. Recently, Bushby and co-workers reported that up to two of the four amino groups in a fully $\mathrm{N}$-aryl-substituted tetraaza[ $\left[1_{4}\right]$ metacyclophane 2 were electrochemically oxidizable. However, the observed oxidation potentials were prohibitively high. Therefore, their attempts to generate the dication of $\mathbf{2}$ by the chemical oxidation ended in failure [17]. Finally, Tsue and co-workers succeeded in generating the spin-triplet di(radical cation) of the $N$-methylsubstituted tetraaza[ $\left.1_{4}\right]$ metacyclophane 3 by protecting the tert-butyl and methoxy groups [18]. However, it still proved impossible to remove the third and fourth electrons. These results indicate that 


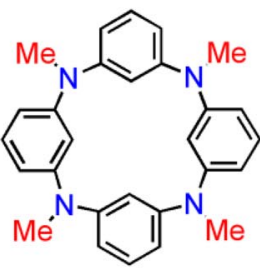

1<smiles>COc1ccccc1N(c1ccccc1)c1cccc(N(c2ccccc2)c2cccc(N(c3ccccc3)c3ccccc3OC)c2)c1</smiles>

2

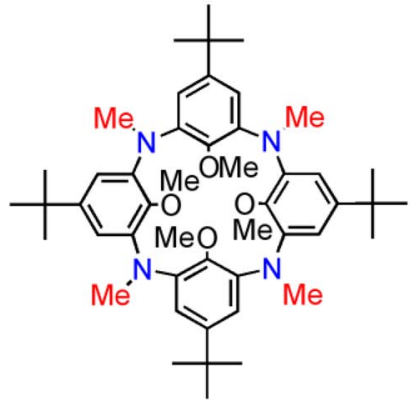

3<smiles>COc1ccc(N(c2ccc(OC)cc2)c2ccc(N(c3cccc(N(c4ccc(OC)cc4)c4ccc(OC)cc4)c3)c3cccc(N(c4cccc(N(c5ccc(OC)cc5)c5ccc(OC)cc5)c4)c4cccc(N(c5ccc(N(c6ccc(OC)cc6)c6ccc(OC)cc6)cc5)c5cccc(N(c6ccc(OC)cc6)c6ccc(OC)cc6)c5)c4)c3)cc2)cc1</smiles>

Fig. 5 Fully $N$-substituted tetraaza $\left[1_{4}\right]$ metacyclophanes $\mathbf{1 - 4}$. The blue-colored moieties correspond to the redoxactive units.

the strong Coulombic interaction among the charged sites hinders generation of the higher oxidation states of tetraaza[1 $\left.1_{4}\right]$ metacyclophane [17].

To alleviate the Coulombic penalty and to lower the oxidation potentials, the introduction of dianisylaminophenyl groups at all of the $\mathrm{N}$-positions is a useful strategy. This modification leads to stabilization of the generated poly(radical cation)s due to the spin delocalization over the para-PD moieties, as well as lowering the oxidation potentials due to the electron-donating ability of the dianisylamino group. To test this theory, we have prepared 4-( $N, N$-dianisylamino)phenyl-substituted tetraaza $\left[1_{4}\right]$ metacyclophane 4 [12].

Electrochemical oxidation of the macrocycle 4 by the cyclic voltammetry in dichloromethane showed four redox couples, and on the basis of the potential-controlled coulometry, each oxidation process was found to correspond to a two-electron oxidation. Table 1 shows that the oxidation potentials of the macrocycle 4 are greatly reduced as compared with those of the reported aza[ $\left.1_{4}\right]$ metacyclophanes 1-3. Hence, macrocycle 4 was found to be oxidizable up to dication, tetracation, hexacation, and octacation. From the comparison of the redox-potentials of the tetraanisyl-p-phenylenediamine $\left[E_{1}{ }^{\mathrm{ox}}=\right.$ $-0.13 \mathrm{~V} ; E_{2}{ }^{\mathrm{ox}}=0.35 \mathrm{~V}$ vs. $\mathrm{Fc}^{0 /+}$ under the same conditions], two electrons are removed simultaneously from the two diagonally opposite PD moieties to reduce the Coulombic repulsion between the charged semi-quinoid PD moieties in the first oxidation process. Subsequently, the remaining two neutral PD moieties in $4^{2+}$ are oxidized simultaneously into tetra(radical cation) $4^{4+}$ in the second oxidation process. 
Table 1 The oxidation potential ${ }^{\mathrm{a}}\left(\mathrm{V}\right.$ vs. $\mathrm{Fc}^{0 /+}$ ) of

1-4.

\begin{tabular}{lcccc}
\hline Compound & $E_{1}{ }^{\text {ox }}$ & $E_{2}{ }^{\text {ox }}$ & $E_{3}{ }^{\text {ox }}$ & $E_{4}{ }^{\text {ox }}$ \\
\hline $\mathbf{1}$ & $0.31^{\mathrm{b}}$ & - & - & - \\
$\mathbf{2}$ & $0.79^{\mathrm{c}}$ & $0.97^{\mathrm{c}}$ & - & - \\
$\mathbf{3}$ & $0.14^{\mathrm{d}}$ & $0.45^{\mathrm{d}}$ & - & - \\
$\mathbf{4}$ & $-0.05^{\mathrm{e}}$ & $0.02^{\mathrm{e}}$ & $0.44^{\mathrm{e}}$ & $0.57^{\mathrm{e}}$ \\
\hline
\end{tabular}

${ }^{\mathrm{a}} 0.1 \mathrm{M} n-\mathrm{Bu}_{4} \mathrm{NBF}_{4}$ in $\mathrm{CH}_{2} \mathrm{Cl}_{2}$ at rt.

${ }^{\mathrm{b}}$ Anodic peak potential (irreversible), see ref. [16].

${ }^{c} n$-Bu $\mathrm{NBF}_{4}$ was replaced by $n-\mathrm{Bu}_{4} \mathrm{NPF}_{6}$, see ref. [17].

dSee ref. [18].

eTwo-electron oxidation, see ref. [12].

To gain the corroborative evidence for the spin-multiplicity of $\mathbf{4}^{2+}$ and $\mathbf{4}^{4+}$ at low temperatures, we have measured the electron spin transient nutation (ESTN) spectra on the basis of the pulsed electron spin resonance (ESR) method [19]. The ESTN method is based on the fact that magnetic moments with different spin multiplicities precess with a specific nutation frequency $\left(\omega_{\text {nut }}\right)$ in the presence of a microwave irradiation field and a static magnetic field. When the Zeeman $H_{Z}$ and fine-structure $H_{D}$ terms are assumed for high-spin systems with spin quantum number $S$, the spin Hamiltonian is given as

$$
\boldsymbol{H}=H_{Z}+H_{D}=-\gamma_{e} \hbar B_{0} S_{z}+\boldsymbol{S} \cdot \boldsymbol{D} \cdot \boldsymbol{S}
$$

where $\gamma_{e}$ is the gyromagnetic ratio of electron, and $\boldsymbol{D}$ is fine-structure tensor. Here, the static magnetic field $B_{0}$ is applied along to the laboratory $z$-axis, and furthermore, the $g$-tensor is assumed isotropic. When the microwave irradiation field $B_{1}$ perpendicular to the magnetic field $B_{0}$, the rotating-frame Hamiltonian can be represented by

$$
\boldsymbol{H}_{R}=H_{D}-\gamma_{e} \hbar B_{1} S_{x}
$$

where the resonance offset term is neglected (the Zeeman term disappears from the description).

All the transition frequencies between eigenstates of $\boldsymbol{H}_{R}$ are regarded as nutation frequencies. Using the density matrix formalism [20], the nutation frequencies can be determined by projecting the eigenstates of $\boldsymbol{H}_{R}$ onto the eigenstates of $\boldsymbol{H}$ as a function of the ratio of $\left|H_{D}\right|$ and $\left|\gamma_{e} \hbar B_{1} S_{x}\right|$. Without complicated calculations, useful pictures are easily obtained for two extreme cases: $\left|H_{D}\right|<<\left|\gamma_{e} \hbar B_{1} S_{x}\right|$ and $\left|\gamma_{e} \hbar B_{1} S_{x}\right|<<\left|H_{D}\right|$. In the first case, $H_{D}$ is negligible to $-\gamma_{e} \hbar B_{1} S_{x}$ and hence, the nutation frequency is simply $\gamma_{e} B_{1}=\omega_{1}$, which is independent of spin quantum numbers $S$ and $M_{S}$ and therefore corresponds to the nutation frequency for the non-interacting spin-doublet system. In the second case, $-\gamma_{e} \hbar B_{1} S_{x}$ term can be considered to be a perturbation to $H_{D}$ term and therefore, an eigenstate of $\boldsymbol{H}_{R}$ can be written as a linear combination of the eigenstates of $H_{D}$, which are characterized by the same magnetic quantum number $M_{S}$ of $\boldsymbol{H}$. From the eigenvalue equation for two states $\left|S, M_{S}\right\rangle$ and $\left|S, M_{S}-1\right\rangle$ :

$$
\begin{aligned}
& \left(\begin{array}{cc}
\left\langle S, M_{S}\left|-\hbar \omega_{1} S_{x}\right| S, M_{S}\right\rangle-E & \left\langle S, M_{S}\left|-\hbar \omega_{1} S_{x}\right| S, M_{S}-1\right\rangle \\
\left\langle S, M_{S}-1\left|-\hbar \omega_{1} S_{x}\right| S, M_{S}\right\rangle & \left\langle S, M_{S}-1\left|-\hbar \omega_{1} S_{x}\right| S, M_{S}-1\right\rangle-E
\end{array}\right) \\
& =\left(\begin{array}{cc}
-E & -\frac{1}{2} \sqrt{S(S+1)-M_{S}\left(M_{S}-1\right)} \hbar \omega_{1} \\
-\frac{1}{2} \sqrt{S(S+1)-M_{S}\left(M_{S}-1\right)} \hbar \omega_{1} & -E
\end{array}\right)=0
\end{aligned}
$$


an energy splitting of $\Delta E=\sqrt{S(S+1)-M_{S}\left(M_{S}-1\right)} \hbar \omega_{1}$ and thus the nutation frequency $\omega_{\text {nut }}$ for the $\left|S, M_{S}\right\rangle \leftrightarrow\left|S, M_{S}-1\right\rangle$ allowed transition is given by

$$
\omega_{\text {nut }}=\sqrt{S(S+1)-M_{S}\left(M_{S}-1\right)} \omega_{1}
$$

This equation indicates that the nutation frequency $\omega_{\text {nut }}$ can be scaled with the spin quantum numbers $S$ and $M_{\mathrm{S}}$ in a unit of $\omega_{1}$, which is equivalent to the nutation frequency of the spin-doublet systems $\left[\omega_{1}=\omega_{\text {doublet }}\right]$. Hence, the ESTN spectroscopy is considered as a powerful tool to determine the definite spin-multiplicity of the high-spin molecules without the spectral simulations for randomly oriented high-spin systems and to discern the specific high-spin molecule from the mixture of high-spin molecules with different spin-multiplicities [19].

The observed nutation spectra at $5 \mathrm{~K}$ for the dicationic $\left(4^{2+}\right)$ and tetracationic $\left(4^{4+}\right)$ species generated by chemical oxidation are shown in Fig. 6 . The ratios between the observed nutation frequencies $\left(\omega_{\text {nut }}\right)$ are rationalized as the corresponding allowed transitions for the specific spin state with the spin quantum numbers $S$ and $M_{S}$ on the basis of eq. (4). The observed nutation frequencies and their assignments for $\mathbf{4}^{2+}$ and $\mathbf{4}^{4+}$ are summarized in Table 2. This observation therefore unequivocally established that the spin states for $\mathbf{4}^{2+}$ and $\mathbf{4}^{4+}$ were spin-triplet $(S=1)$ and spin-quintet $(S=2)$, respectively. Note that the tetracation $4^{4+}$ was stable at room temperature for several days in solution.

(a)

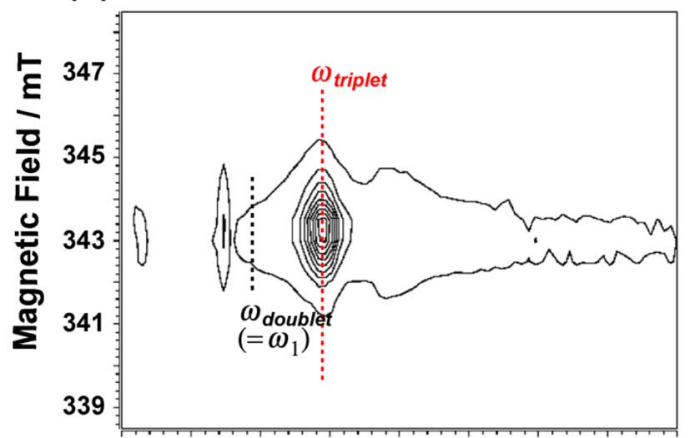

(b)

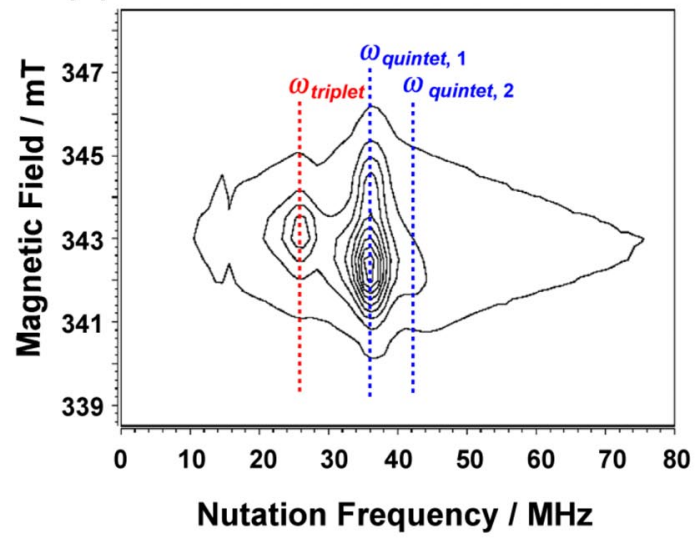

Fig. 6 2D ESTN spectra of (a) $4^{2+}$ and (b) $4^{4+}$ in $n$-butyronitrile at $5 \mathrm{~K}$. 
Table 2 Spectral data of the 2D-ESTN spectroscopy for $4^{2+}$ and $4^{4+}$.

\begin{tabular}{lcl}
\hline Species & Obs. nutation freq./MHz & Transition assignment \\
\hline $\mathbf{4}^{2+}$ & $29\left(\simeq \sqrt{2} \omega_{\text {doublet }}\right)$ & $|1, \pm 1\rangle \Leftrightarrow|1,0\rangle$ \\
(doublet impurity & $20\left(=\omega_{\text {doublet }}\right)$ & $|1 / 2,+1 / 2\rangle \Leftrightarrow|1 / 2,-1 / 2\rangle)$ \\
$\mathbf{4}^{4+}$ & $36\left(\simeq \sqrt{2} \omega_{\text {triplet }}\right)$ & $|2, \pm 2\rangle \Leftrightarrow|2, \pm 1\rangle$ \\
& $\sim 44\left(\simeq \sqrt{3} \omega_{\text {triplet }}\right)$ & $|2, \pm 1\rangle \Leftrightarrow|2,0\rangle$ \\
(competing triplet & $26\left(=\omega_{\text {triplet }}\right)$ & $|1, \pm 1\rangle \Leftrightarrow|1,0\rangle)$ \\
\hline
\end{tabular}

\section{Trimacrocyclic arylamine}

Tetraazacyclophanes $\mathbf{5 a}$ and $\mathbf{5 b}$ shown in Fig. 7 are considered to be the smallest macrocycle having the alternating meta-para linkage [21]. We clarified this molecule can be oxidized into di(radical cation) with triplet spin multiplicity. Afterwards, Hartwig succeeded in synthesizing 1D ladder-like extended molecular systems of the tetraazacyclophane [22]. However, the generated dication of the three-runged ladder molecule resulted in the spin-singlet state. When the tetraazacyclophane $\mathbf{5 b}$ is utilized as a building block for constructing polymacrocyclic molecular systems, the star-shaped extension is promising for realization of $2 \mathrm{D}$ polymer. In this context, we have prepared the star-shaped trimacrocycle 8 [13].
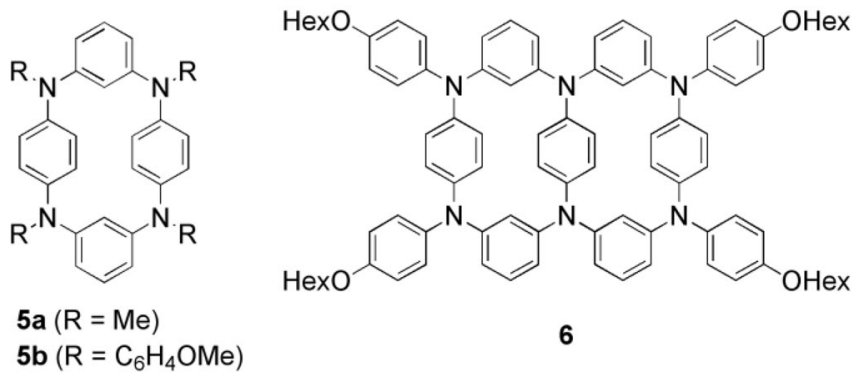

6

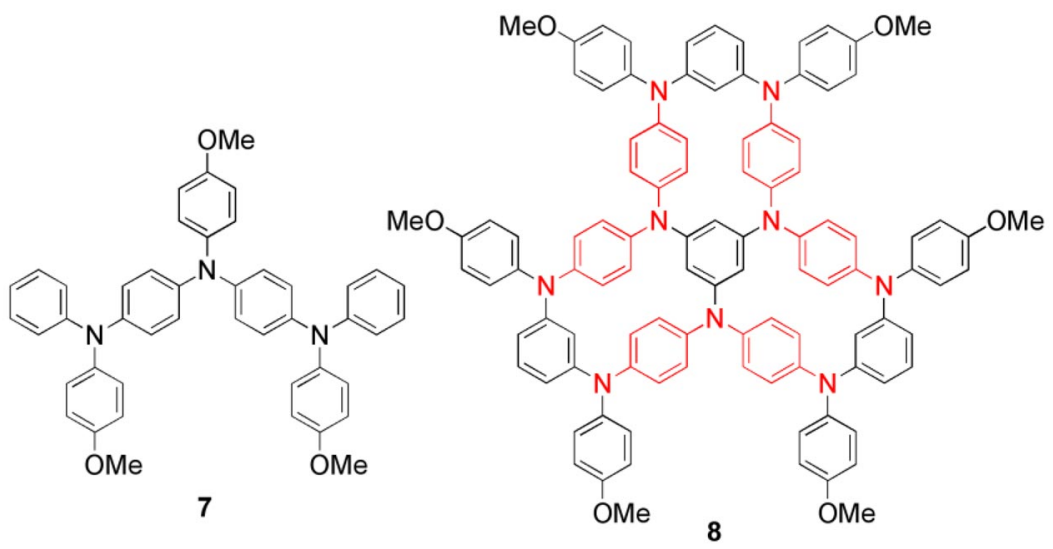

Fig. 7 Tetraaza[ $\left.1_{4}\right] m, p, m, p$-cyclophane $(\mathbf{5 a}, \mathbf{b})$, its polymacrocyclic extended compounds $(\mathbf{6}$ and $\mathbf{8})$ and the related compound $\mathbf{7}$ as the reference for $\mathbf{8}$.

Electrochemical oxidation of the trimacrocycle $\mathbf{8}$ by the cyclic voltammetry showed five reversible redox couples, and the final oxidation process was found to correspond to quasi-two-electron transfer. Hence, the trimacrocycle $\mathbf{8}$ is oxidizable up to hexacation. The oxidation potentials of $\mathbf{8}$ are 
summarized in Table 3 together with those of the reference compounds $\mathbf{5 b}$ and $\mathbf{7}$ (Fig. 7). As compared with the oxidation potentials for the reference compounds, the first three redox couples correspond to electron removal from the three triamine moieties, which are shown in red in Fig. 7.

Table 3 The oxidation potential ${ }^{\mathrm{a}}\left(\mathrm{V}\right.$ vs. $\left.\mathrm{Fc}^{\mathrm{0} /+}\right)$ of $\mathbf{5 b}, \mathbf{7}$, and $\mathbf{8}$.

\begin{tabular}{lccccc}
\hline Compound & $E_{1}{ }^{\text {ox }}$ & $E_{2}{ }^{\text {ox }}$ & $E_{3}{ }^{\text {ox }}$ & $E_{4}{ }^{\text {ox }}$ & $E_{5}{ }^{\text {ox }}$ \\
\hline $\mathbf{5 b}$ & -0.01 & 0.22 & 0.54 & 0.67 & - \\
$\mathbf{7}$ & -0.11 & 0.25 & $0.80^{\mathrm{b}}$ & - & - \\
$\mathbf{8}$ & -0.12 & 0.09 & 0.28 & 0.41 & $0.53^{\mathrm{c}}$ \\
\hline
\end{tabular}

${ }^{\mathrm{a}} 0.1 \mathrm{M} \mathrm{n}-\mathrm{Bu}_{4} \mathrm{NBF}_{4}$ in $\mathrm{CH}_{2} \mathrm{Cl}_{2}$ at rt, see ref. [4].

${ }^{\mathrm{b}}$ Anodic peak potential (irreversible).

${ }^{\mathrm{c}}$ Two-electron oxidation.

To identify the spin-multiplicitiy of the oxidized trimacrocycle $\mathbf{8}$, we again carried out the pulsed ESR measurements for dicationic $\left(\mathbf{8}^{2+}\right)$ and tricationic $\left(\mathbf{8}^{3+}\right)$ species of the trimacrocycle $\mathbf{8}$. The ESTN spectra at $5 \mathrm{~K}$ for $\mathbf{8}^{2+}$ and $\mathbf{8}^{3+}$ generated by chemical oxidation are shown in Fig. 8 . The observed nutation frequencies and their assignments based on eq. 4 are summarized in Table 4 . This observation therefore unequivocally established that the spin states for $\mathbf{8}^{2+}$ and $\mathbf{8}^{3+}$ were spin-triplet $(S=1)$ and spin-quartet $(S=3 / 2)$, respectively. Consequently, the high-spin alignment for the generated dication and trication of the trimacrocycle $\mathbf{8}$ was achieved at low temperatures. Moreover, the trication $\mathbf{8}^{3+}$ was stable at room temperature for a few days in solution.

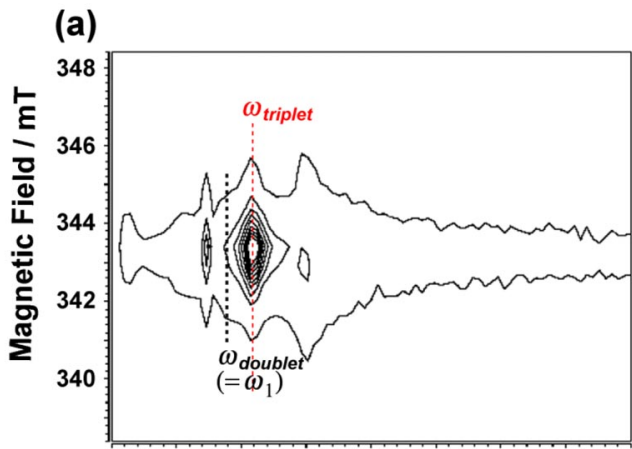

(b)

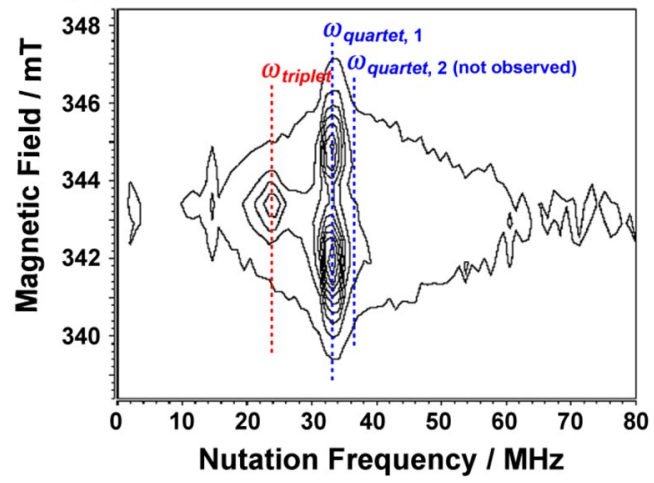

Fig. 8 2D ESTN spectra of (a) $8^{2+}$ and (b) $8^{3+}$ in $n$-butyronitrile at $5 \mathrm{~K}$. 
Table 4 Spectral data of the 2D-ESTN spectroscopy for $\mathbf{8}^{2+}$ and $\mathbf{8}^{3+}$.

\begin{tabular}{lcl}
\hline Species & Obs. nutation freq./MHz & Transition assignment \\
\hline $\mathbf{8}^{2+}$ & $22\left(\simeq \sqrt{2} \omega_{\text {doublet }}\right)$ & $|1, \pm 1\rangle \Leftrightarrow|1,0\rangle$ \\
(doublet impurity & $18\left(\omega_{\text {doublet }}\right)$ & $|1 / 2,+1 / 2\rangle \Leftrightarrow|1 / 2,-1 / 2\rangle)$ \\
$\mathbf{8}^{3+}$ & $33\left(\simeq \sqrt{3 / 2} \omega_{\text {triplet }}\right)$ & $|3 / 2, \pm 3 / 2\rangle \Leftrightarrow|3 / 2, \pm 1 / 2\rangle$ \\
(triplet impurity & $24\left(\omega_{\text {triplet }}\right)$ & $|1, \pm 1\rangle \Leftrightarrow|1,0\rangle)$ \\
\hline
\end{tabular}

\section{CONCLUSION}

We demonstrated that the present multi-spin system, which was generated from the tetraazacyclophane 4 or the trimacrocycle 8, exhibited high-spin preference. The all- $N$-substitution of tetraaza[ $\left.1_{4}\right]$ metacyclophane with dianisylaminophenyl groups was able to avoid the Coulombic penalty which often led to the difficulty in generating higher oxidation states of oligoarylamines, and this modification is considered to be a useful approach to stabilize the dication and tetracation. On the other hand, the starshaped extension of alternating meta-para tetraazacyclophane was also found to be a useful strategy to stabilize the high-spin state, as compared with the ladder-shaped extension. Furthermore, recently, we have found that the high-spin alignment in oligoarylamine-based multi-spin system can also be strengthened by incorporating cyclophane skeletons into the oligomer backbone [23]. In addition to the quest for the suitable linkage of polymacrocyclic oligoarylamines, the search for a more versatile synthetic methodology of the macrocyclic oligoarylamines is also important. In this line, there is ongoing active development [24-28].

The present multi-spin molecular systems based on macrocyclic oligoarylamines may afford the possibility for the extension to larger 2D molecular spin systems, and hence, further studies on the extended molecular systems based on $\mathbf{4}$ and $\mathbf{8}$ are still underway.

\section{SUPPLEMENTARY INFORMATION}

Supplementary information is available online (doi:10.1351/PAC-CON-09-10-16).

\section{ACKNOWLEDGMENTS}

The authors acknowledge Dr. Ko Furukawa at the Institute for Molecular Science and Prof. Tatsuhisa Kato at the Department of Chemistry, Josai University for the pulsed ESR measurements. Thanks are due to the Instruments Center, the Institute for Molecular Science for use of the pulsed ESR spectrometer. The present work was supported by Grant-in-Aid for Scientific Research (B) (20350065) from Japan Society for the Promotion of Science (JSPS).

\section{REFERENCES}

1. (a) N. Mataga. Theor. Chim. Acta 10, 372 (1968); (b) K. Itoh. Pure Appl. Chem. 50, 1251 (1978).

2. N. D. Mermin, H. Wagner. Phys. Rev. Lett. 17, 1133 (1966).

3. A. Rajca. Chem.-Eur. J. 8, 4834 (2002) and refs. therein.

4. (a) Y.-T. Wu, J. S. Siegel. Chem. Rev. 106, 4843 (2006); (b) V. M. Tsefrikas, L. T. Scott. Chem. Rev. 106, 4868 (2006).

5. A. Rajca, S. Rajca, S. R. Desai. J. Am. Chem. Soc. 117, 806 (1995).

6. K. Matsuda, N. Nakamura, K. Takahashi, K. Inoue, N. Koga, H. Iwamura. J. Am. Chem. Soc. 117, 5550 (1995). 
7. (a) A. Ito, K. Ota, K. Tanaka, T. Yamabe, K. Yoshizawa. Macromolecules 28, 5618 (1995); (b) A. Ito, T. Saito, K. Tanaka, T. Yamabe. Tetrahedron Lett. 36, 8809 (1995); (c) A. Ito, T. Saito, K. Ota, T. Miura, Y. Misaki, K. Tanaka, T. Yamabe. J. Mater. Chem. 8, 1799 (1998); (d) A. Ito, H. Ino, K. Tanaka, K. Kanemoto, T. Kato. J. Org. Chem. 67, 491 (2002).

8. (a) J. P. Wolfe, S. Wagaw, J.-F. Marcoux, S. L. Buchwald. Acc. Chem. Res. 31, 805 (1998); (b) J. F. Hartwig. Acc. Chem. Res. 31, 852 (1998); (c) J. F. Hartwig. Angew. Chem., Int. Ed. 37, 2046 (1998); (d) A. R. Muci, S. L. Buchwald. Top. Curr. Chem. 219, 131 (2002).

9. M. M. Wienk, R. A. J. Janssen. J. Am. Chem. Soc. 119, 4492 (1997).

10. A. Ito, D. Sakamaki, H. Ino, A. Taniguchi, Y. Hirao, K. Tanaka, K. Kanemoto, T. Kato. Eur. J. Org. Chem. 4441 (2009) and refs. therein.

11. A. Ito, H. Ino, K. Tanaka. Polyhedron 28, 2080 (2009).

12. A. Ito, S. Inoue, Y. Hirao, K. Furukawa, T. Kato, K. Tanaka. Chem. Commun. 3242 (2008).

13. A. Ito, Y. Yamagishi, K. Fukui, S. Inoue, Y. Hirao, K. Furukawa, T. Kato, K. Tanaka. Chem. Commun. 6573 (2008).

14. G. W. Smith. Nature 198, 879 (1963).

15. For the recent review on azacalixarene, see: H. Tsue, K. Ishibashi, R. Tamura. Top. Heterocycl. Chem. 17, 73 (2008).

16. (a) A. Ito, Y. Ono, K. Tanaka. New J. Chem. 779 (1998); (b) A. Ito, Y. Ono, K. Tanaka. J. Org. Chem. 64, 8236 (1999).

17. R. J. Bushby, C. A. Kilner, N. Taylor, M. E. Vale. Tetrahedron 63, 11458 (2007).

18. K. Ishibashi, H. Tsue, N. Sakai, S. Tokita, K. Matsui, J. Yamauchi, R. Tamura. Chem. Commun. 2812 (2008).

19. (a) J. Isoya, H. Kanda, J. R. Norris, J. Tang, M. K. Bowman. Phys. Rev. B 41, 3905 (1990); (b) A. V. Astashkin, A. Schweiger. Chem. Phys. Lett. 174, 595 (1990); (c) K. Sato, M. Yano, M. Furuichi, D. Shiomi, T. Takui, K. Abe, K. Itoh, A. Higuchi, K. Katsuma, Y. Shirota. J. Am. Chem. Soc. 119, 6607 (1997); (d) A. Ito, H. Ino, K. Tanaka, K. Kanemoto, T. Kato. J. Org. Chem. 67, 491 (2002).

20. T. Farrar, J. E. Harriman. Density Matrix Theory and Its Application in NMR Spectroscopy, The Farragut Press, Madison (1992).

21. (a) A. Ito, Y. Ono, K. Tanaka. Angew. Chem., Int. Ed. 39, 1072 (2000); (b) T. D. Selby, S. C. Blackstock. Org. Lett. 1, 2053 (1999); (c) S. I. Hauck, K. V. Lakshmi, J. F. Hartwig. Org. Lett. 1, 2057 (1999); (d) I. Kulszewicz-Bajer, V. Maurel, S. Gambarelli, I. Wielgus, D. Djurado. Phys. Chem. Chem. Phys. 11, 1362 (2009).

22. X. Z. Yan, J. Pawlas, T. Goodson III, J. F. Hartwig. J. Am. Chem. Soc. 127, 9105 (2005).

23. D. Sakamaki, A. Ito, Ko Furukawa, T. Kato, K. Tanaka. Chem. Commun. 4524 (2009).

24. W. Fukushima, T. Kanbara, T. Yamamoto. Synlett 2931 (2005).

25. K. Ishibashi, H. Tsue, S. Tokita, K. Matsui, H. Takahashi, R. Tamura. Org. Lett. 8, 5991 (2006).

26. M. Touil, M. Lachkar, O. Siri. Tetrahedron Lett. 49, 7250 (2008).

27. M. Vale, M. Pink, S. Rajca, A. Rajca. J. Org. Chem. 73, 27 (2008).

28. H. Konishi, S. Hashimoto, T. Sakakibara, S. Matsubara, Y. Yasukawa, O. Morikawa, K. Kobayashi. Tetrahedron Lett. 50, 620 (2008). 